\title{
Reflets
}

Revue d'intervention sociale et communautaire

\section{De la conformité au refus : analyse d'un parcours}

\section{Jean-Luc Pinard et Nérée St-Amand}

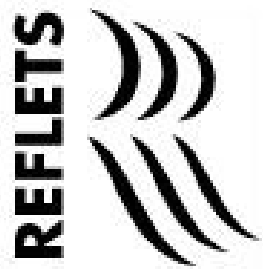

Volume 19, numéro 1, printemps 2013

Approches structurelles et intervention sociale

URI : https://id.erudit.org/iderudit/1018043ar

DOI : https://doi.org/10.7202/1018043ar

Aller au sommaire du numéro

Éditeur(s)

Reflets, Revue d'intervention sociale et communautaire

ISSN

1203-4576 (imprimé)

1712-8498 (numérique)

Découvrir la revue

Citer cet article

Pinard, J.-L. \& St-Amand, N. (2013). De la conformité au refus : analyse d'un parcours. Reflets, 19(1), 85-118. https://doi.org/10.7202/1018043ar

\section{Résumé de l'article}

Dans le contexte d'une recherche menée auprès depersonnes ayant vécu des expériences en psychiatrie auNouveau-Brunswick, cet article propose une réflexion surcertaines stratégies de débrouillardiseprivilégiées par l'une d'entre elles àla suite d'un diagnostic de maladie mentale. Une grillestructurelle permettra de comprendre certains des enjeux enprésence tandis que sur le plan méthodologique,l'analyse d'un parcours permettra de mettre en valeur uneétude de cas comme illustration de possiblesréalités émergentes.
Tous droits réservés (C) Reflets, Revue d'intervention sociale et communautaire, 2013 cest protégé par la loi sur le droit d'auteur. L'utilisation des services d'Érudit (y compris la reproduction) est assujettie à sa politique d'utilisation que vous pouvez consulter en ligne. 


\section{De la conformité au refus : analyse d'un parcours}

Jean-Luc Pinard

Candidat au doctorat - École de service social, Université d'Ottawa

Nérée St-Amand

Professeur titulaire - École de service social, Université d'Ottawa

\section{Résumé}

Dans le contexte d'une recherche menée auprès de personnes ayant vécu des expériences en psychiatrie au Nouveau-Brunswick, cet article propose une réflexion sur certaines stratégies de débrouillardise privilégiées par l'une d'entre elles à la suite d'un diagnostic de maladie mentale. Une grille structurelle permettra de comprendre certains des enjeux en présence tandis que sur le plan méthodologique, l'analyse d'un parcours permettra de mettre en valeur une étude de cas comme illustration de possibles réalités émergentes.

Mots clés : Oppression, approche structurelle, pratiques opprimantes, institutions psychiatriques, violence institutionnelle, désinstitutionnalisation, antipsychiatrie, stigmatisation, reprise de pouvoir, étude de cas, expérience narrative, personnes psychiatrisées, pouvoir psychiatrique, reconstruction de soi.

\section{Abstract}

In the context of a New Brunswick research project involving people having had experiences with the psychiatric system, this article highlights some coping strategies used by one of the people interviewed after she was diagnosed as mentally ill. A structural analysis helped us to analyse and appreciate some of the variables 
in her determined efforts to move away from the psychiatric system while, at the methodological level, this case study reveals and illustrates possible emerging realities within the ex-psychiatric movement.

Key words : Oppression, structural approach, oppressive practices, psychiatric institutions, institutional violence, deinstitutionalization, antipsychiatry, stigma, empowerment, case study, narrative experience, psychiatric survivors, psychiatric expatients, psychiatric power, self rebuilding.

\section{Introduction}

"Les données

recueillies à l'aide de

31 entrevues révélaient une présence persistante de l'institution et de ses experts dans la vie quotidienne des personnes..."

"...elle refuse de continuer à jouer un jeu qui l'a même conduite aux soins intensifs."
Au début des années 2000, nous avons entrepris de dresser un portrait de la réalité des personnes ayant connu différentes expériences d'internement psychiatrique au Nouveau-Brunswick francophone $^{1}$, à la suite de la vague de désinstitutionnalisation que cette province avait connue depuis les années 1970. Les données recueillies à l'aide de 31 entrevues révélaient une présence persistante de l'institution et de ses experts dans la vie quotidienne des personnes, même si les lieux et les modalités de prise en charge avaient fait l'objet de certaines transformations (Pinard et St-Amand, 2012). Notre constat initial a donc été d'observer l'emprise continue et complexe de l'institution et des professionnels sur les personnes psychiatrisées.

Par ailleurs, nous avons rencontré une personne qui faisait nettement figure d'exception à cette tendance. Il s'agit de Sylvie (nom fictif), une jeune femme qui, soumise pendant plusieurs années à un diagnostic menant à des traitements psychiatriques, constate les dommages que lui causent tant la psychiatrie que l'ensemble du réseau d'aide professionnelle. Elle entreprend alors une démarche de reprise en charge de sa vie, de réappropriation de son destin qu'elle avait pendant des années confié aux soins des services de santé mentale. Après quelques tentatives de négociations et constatant que la psychiatrie est en train de ruiner sa vie, elle refuse de continuer à jouer un jeu qui l'a même conduite aux soins intensifs. Elle entreprend alors de refaire sa 
vie en explorant diverses approches alternatives, faisant preuve du même coup d'une grande capacité de résilience.

Psychiatre, ergothérapeute et gestionnaires de dossier représentent à ses yeux les acteurs principaux d'une structure opprimante qui aura aggravé son état de santé, ruiné ses projets de vie et hypothéqué sa condition médicale et sociale. L'indignation et l'énergie du refus forment la trame de son récit de vie. Il s'agit d'une situation porteuse de plusieurs questionnements, lorsqu'analysés grâce à une analyse structurelle et antipsychiatrique.

\section{L'étude de cas comme illustration d'une problématique sociale}

"...qu'une telle

méthode a le mérite

de montrer le nonsens d'une psychiatrie axée sur le diagnostic et le traitement des symptômes..."
Après avoir étudié le récit initial de Sylvie, nous avons décidé de l'approfondir et d'en faire une étude de cas; cette dernière est tout indiquée pour comprendre les nuances et méandres du parcours de la personne en ce qu'elle " permet une compréhension profonde des phénomènes, des processus les composant et des personnes y prenant part» (Gagnon, 2005, p. 2). Il s'agit alors de mettre l'accent sur une optimisation de la compréhension de l'expérience de l'individu plutôt que sur la généralisation des résultats. Dans le contexte qui nous concerne, nous constaterons qu'une telle méthode a le mérite de montrer le non-sens d'une psychiatrie axée sur le diagnostic et le traitement des symptômes plutôt que sur les besoins réels de la personne ainsi que sur son potentiel de s'en sortir.

«Penser par cas " constitue une méthode spécifiquement adaptée à la situation que nous venons de décrire. Le cas, c'est ce qui vient déranger, provoquer un raisonnement différent par sa singularité ou sa différence (Passeron, 2005). Même s'il ne fait pas preuve, le cas contribue à faire obstacle aux généralisations et prétentions de données probantes, notamment dans le contexte de la domination du savoir biomédical. Grâce à une telle méthodologie, l'accent est mis sur le potentiel de la personne plutôt que sur la variété des informateurs, la capacité 
de renseigner d'un cas étant ici privilégiée à sa représentativité (Gagnon, 2005, p. 46). Cette méthode de recherche comporte un potentiel d'apport en connaissances riches et inédites, visant à approfondir la compréhension des résultats plutôt que leur généralisation : «Ses grandes forces sont bien sûr de fournir une analyse en profondeur des phénomènes dans leur contexte, d'offrir la possibilité de développer des paramètres historiques, d'assurer une forte validité interne. " (Gagnon, 2005, p. 3)

Demander aux gens de décrire leur expérience en psychiatrie, c'est leur offrir un espace de parole et permettre en même temps au chercheur d'identifier et d'analyser des actes ou des événements significatifs ayant marqué l'existence de la personne. Face au chercheur qui ne connait ni l'individu ni son milieu, l'informateur construit, à partir de moments choisis, des éléments qui l'ont marqué, qui ont forgé son identité. Il s'agit de l'étude de cas fondée sur l'approche autobiographique (Pineau et MarieMichèle, 1983; Pineau et Le Grand, 1993) : « [...] Une vie peut ne pas être seulement une simple chronique, une succession de faits divers s'empilant selon une chronologie à causalité linéaire, mais un drame historique se nouant récursivement et prospectivement autour d'événements." (Cité de Catani [1978] dans Pineau et Marie-Michèle, 1983, p. 175)

Pineau et Marie-Michèle (1983) attribuent à Jean-Paul Sartre le mérite d'avoir donné un statut épistémologique à part entière à l'existence, au vécu individuel, à l'expérience singulière, à ce que ce dernier appelle " le mouvement dialectique originel dans l'individu et dans son entreprise de produire sa vie, de s'objectiver " (p. 160). En somme, le social se retrouve dans l'individu et ce dernier peut analyser sa réalité et démontrer comment il est personnellement affecté par les pressions sociales, les normes, les jugements de la société à son égard. Cette existence prend aussi son importance dans la pensée des pionniers de l'antipsychiatrie que sont David Cooper et Ronald J. Lang, dans ce que Sartre appellera une approche "existentielle" des malades mentaux : "Je pense comme vous qu'on ne peut comprendre les troubles psychiques du dehors, à partir du déterminisme positiviste ni les reconstruire par une combinaison de concepts qui restent 
extérieurs à la maladie vécue. » (Préface de J.-P. Sartre, dans Laing et Cooper, 1971)

L'approche autobiographique permet d'atteindre trois types de finalités, soit l'agir, le comprendre et l'émancipation. "Pour être utile à la vie, l'histoire doit être critique, éclairant et renforçant le présent »(Pineau et Marie-Michèle, 1983, p. 75). Ainsi, à partir de sa narration, on peut apprendre comment le sujet a pu se retrouver dans tel type de situation, distinctement de toute interprétation médicale, professionnelle ou autre. En fait, "les histoires sont souvent plus intéressantes et persuasives que les analyses statistiques. En plus d'être plus faciles à mémoriser, elles rendent mieux compte de la complexité que de minces descriptions neutres " (Gagnon, 2005, p. 112).

Cette approche implique à la fois une sélection des faits et leur interprétation par la personne, étant entendu que la réalité sociale à l'étude doit tenir compte des subjectivités et des intentionnalités de l'individu. Il s'agit donc pour le chercheur de s'ouvrir à de nouvelles façons de voir : il entre dans le milieu étudié avec

"...la réalité sociale à l'étude doit tenir compte des subjectivités et des intentionnalités de l'individu. » une question à portée générale qui se précisera et prendra sa forme définitive grâce au témoignage de l'informateur. Le récit autobiographique traduit ainsi une réalité sociale, en mettant l'accent sur les processus sociaux dans l'expérience de l'individu plutôt que sur son fonctionnement personnel.

\section{L'histoire de Sylvie « l'Importée »}

En avril 2001, alors que nous entreprenions une recherche sur la désinstitutionnalisation et recueillions des témoignages de personnes psychiatrisées, Sylvie se présente à nous, référée par le directeur d'un centre d'activités. Dès cette première rencontre, elle évoque son parcours difficile entre la psychiatrie, l'hôpital et les ressources de santé mentale. Quelques mois plus tard, nous la rencontrons à nouveau afin d'approfondir son témoignage. Sylvie décrit alors ses démarches vers une vie plus autonome, plus assumée. Enfin, nous l'invitons à partager son récit de vie avec un groupe d'étudiants, récit qui met en lumière la débrouillardise de 
la jeune femme et la maturité de sa prise en charge. Retranscrits, ces trois entretiens constituent le matériel de base de la présente étude de cas. D’abord, histoire de Sylvie.

\section{« L'Importée »}

Née au Nouveau-Brunswick d'un parent québécois et d'un parent issu d'une autre ethnie, Sylvie s'est vue affublée enfant du sobriquet de "l'Importée" (sobriquet fictif aussi, mais qui reproduit le caractère volontairement offensant de celui qu'elle a eu à endurer). Sylvie a vécu une enfance difficile, éprouvant des difficultés à socialiser, étant l'objet de harcèlement et victime d'attitudes méprisantes de la part des jeunes de son milieu : «Tu devrais retourner dans ton pays! », dit-on à celle qui est pourtant native d'Acadie. Malgré la discrimination dont elle est victime, elle termine ses études secondaires, puis son baccalauréat. Et même si elle se sent fragilisée par des années de taxage, elle part étudier à l'étranger, puis revient travailler au Nouveau-Brunswick. Après avoir occupé quelques emplois précaires, elle se retrouve au chômage et retourne habiter chez ses parents. Elle arrive alors dans un milieu empreint de disputes familiales, ses parents étant en instance de séparation. De surcroit, Sylvie subit régulièrement du harcèlement dans son quartier. Elle souligne un événement qui la perturbe profondément : le chien de la famille est frappé intentionnellement par une auto. L'animal devra subir plusieurs interventions longues et coûteuses.

\section{Tout éclate...}

Une suite d'événements perturbateurs, notamment une exacerbation des tensions familiales, provoque sa première crise. Sylvie se retrouvera pour quelques jours dans un centre de crise avant d'aboutir à l'hôpital. C'est le moment de sa prise en charge par le réseau de la santé mentale. Dès lors, Sylvie passe de personne à cliente (St-Amand, 2011,p.135). Pourtant, elle n'est pas dangereuse :

«Il n'y a personne qui a souffert de ce que j'ai fait. Tout ce qu'ils peuvent me reprocher, c'est d'avoir dit des choses un peu bizarres.» 


\section{La construction d'un « cas » en dix minutes}

Dans une spirale de détérioration de ses conditions de vie et de santé, elle franchit le seuil de services qui vont déterminer son parcours de vie pour plusieurs années. C'est la construction d'un cas psychiatrique à partir d'un moment fatidique qu'elle résume comme suit :

"Je me suis retrouvée à voir un psychiatre qui a décidé après dix minutes que j'étais paranoïaque et schizophrène."

"Je me suis retrouvée à voir un psychiatre qui a décidé après dix minutes que j'étais paranoïaque et schizophrène.»

Comme dans plusieurs situations du genre, certains éléments reviennent constamment : une crise, une référence à la psychiatrie, une brève rencontre avec l'expert, et le sort en est jeté : diagnostic et plan de traitement s'ensuivent. Ces moments marquent la vie des gens concernés, comme ils ont marqué celle de Sylvie. Tout alors la précipite vers l'abime.

\section{«J'ai passé deux semaines au lit! »}

Après son bref séjour à l'hôpital, elle est placée dans un foyer de groupe où levers et couchers sont à heures fixes et où la routine quotidienne est des plus réglementées. Interdiction de faire sa lessive, aucune activité sociale ou récréative, siestes obligatoires, "Si tu t'ennuies, il y a voilà l'essence de son quotidien. Le personnel lui suggère : « Si tu toujours le McDo ou le Tim Hortons..." t'ennuies, il y a toujours le McDo ou le Tim Hortons pas loin.»

Sylvie décide alors d'acheter ses propres légumes et de modifier son alimentation. Cela provoque des conflits avec les responsables de la résidence qui, constate-t-elle alors, n'encouragent pas les gens à prendre soin d'eux-mêmes; tout au contraire, certains résidents passent de longues périodes alités.

"J'ai passé deux semaines au lit et puis, de toute évidence, la résidence trouvait ça normal parce qu'on était trois à faire ça. Au bout de deux semaines, j'ai dit :" OK, je sors de mon lit et puis il faut que je m'en aille ou bien je meurs parce que je ne peux pas continuer comme ça. ”" 
"Elle quitte le foyer de soin, déterminée à chercher son propre logement. "
Sylvie constate que le système auquel elle doit s'adapter comporte des contradictions importantes : "C'est là que j'ai commencé à remarquer les absurdités des systèmes. »

Elle quitte le foyer de soin, déterminée à chercher son propre logement. Cela constitue à notre avis un premier moment fort de la reprise en charge de sa vie, là où elle démontre une grande détermination à changer le cours d'une "carrière " qui lui est déjà toute tracée.

\section{De chambre en taudis...}

Sortie de cette résidence, Sylvie ne trouve pas de logement abordable et cohabite avec des gens " peu recommandables ", selon son expression. Elle doit alors déménager à plusieurs reprises.

"J'ai habité avec un gars parce que j'étais désespérée. Dès que les autorités ont su que j'habitais avec quelqu'un, elles ont réduit mon chèque à $400 \$$ par mois et ont décidé de m’enlever ma carte blanche. Ça veut dire que je ne pouvais plus prendre l'autobus... Dès la première semaine, ceci a créé des tensions entre nous.»

Cette cohabitation prend fin après un mois.

«J'ai été vivre dans un autre taudis insalubre et puis là, j’ai décidé de faire une demande de logement subventionné. Ils ont déterminé que ce n'était pas sécuritaire d'habiter là où j'étais. J'ai eu de la chance :j'ai eu un logement subventionné au bout de quelques mois."

\section{«C'est au docteur à décider ça... »}

"Ils t'encouragent à t'inscrire en invalidité permanente..."
Pendant ce temps, Sylvie subit des pressions pour renoncer à tout rêve de réintégrer le marché du travail.

«Ils t'encouragent à t'inscrire en invalidité permanente parce que tu reçois ainsi plus d'argent 
par mois. Ces gens ont le droit de travailler plus d'heures que quelqu'un qui a le potentiel de s'en sortir.»

Elle confie à une gestionnaire de cas à l'aide sociale : "J'espère un jour recommencer à travailler à temps plein. » Et la gestionnaire de répondre : "C'est au docteur à décider ça..."

De plus en plus, Sylvie se retrouve à la merci des professionnels qui, s'appuyant sur le diagnostic qu'on lui a assigné, briment ses rêves et projets. C'est à contrecœur qu'elle prend ses médicaments et se soumet aux directives de l'équipe de santé mentale.

"J'ai remarqué tout de suite qu'ils étaient extrêmement condescendants. Ils nous parlaient comme si on était des bébés. J'étais vraiment résignée à ma condition officielle : tu es une malade mentale et puis c'est tout ce qu'il y a à propos de toi. Ils parlent vraiment juste de tes symptômes ou de tes limites. »

\section{Fixer le vide...}

Sylvie prendra un puissant psychotrope qui affectera sa santé et son quotidien. Elle est réticente à prendre du Zyprexa, médicament reconnu pour entraîner des prises de poids fulgurantes et provoquer le diabète ${ }^{2}$, maladie qui affecte déjà des membres de sa famille :

«[...] c'est quelque chose qui m'a perturbée un peu parce qu'il y a eu une prise de poids très importante et puis ça me stressait. [...] C'était difficile de me concentrer.Avec le Zyprexa,j'étais toujours un peu endormie.»

Durant cette période, elle prendra plus de 30 kilos.

«Avant,je prenais des petites tailles, du huit.Voici une chose que j'ai trouvée dure : auparavant, j'étais admirée pour ma beauté. [...] Je ne me 
"Je passais les trois quarts de mon temps à fixer le vide..." "...elle refuse que les autres décident de sa vie. » souviens vraiment pas des années 2006 et 2007, j'étais trop droguée. Je passais les trois quarts de mon temps à fixer le vide, j'étais complètement incohérente, je n'arrivais pas à avoir une conversation normale, à trouver mes mots. »

Sylvie fait une embolie pulmonaire ${ }^{3}$ et connait d'autres complications graves de santé. Elle prend aussi du Yaz ${ }^{4}$, un contraceptif dangereux, selon Santé Canada ${ }^{5}$. Les dommages cumulatifs entraînent une hospitalisation et Sylvie passera plus d'une semaine aux soins intensifs : "J'ai failli mourir. Ils m'ont mise sur du Coumadin. [...] Puis, j'ai eu des problèmes digestifs épouvantables." Pour les pallier, elle prend des doses massives d'aspirine. Par la suite, la prise de clozapine ${ }^{6}$ endommage son cœur et son foie.

Elle décide alors de se libérer au plus vite de ces traitements qui risquent de la tuer. Elle passe à une seconde étape de reprise en charge de sa vie : elle refuse que les autres décident de sa vie. En effet, depuis son entrée dans le système psychiatrique, Sylvie avait constaté qu'elle perdait tout pouvoir sur sa vie : toutes les décisions étaient prises à sa place. Qui plus est, au fil des jours, la coercition, selon elle, devenait plus évidente. À force de négociations pénibles - démarches d'ailleurs désapprouvées par l'équipe de soins - elle gagne du terrain sans toutefois se voir reconnue dans son droit au consentement.

"J'essayais de leur expliquer que c'est moi qui prenais ces médicaments-là; c'est moi qui subissais les conséquences. Ils ne voyaient pas ça. Ils pensaient vraiment être l'autorité; ce sont eux qui doivent décider pour toi parce qu'une fois que tu entres en psychiatrie c'est comme si tu avais abandonné le contrôle ou que tu acceptais de laisser quelqu'un d'autre décider pour toi.»

Sylvie remarque également ce qu'elle décrit comme un manque d'éthique au sein du réseau : 
"Je me suis rendu compte qu'il y a beaucoup de gens que je n'avais jamais consultés, mais qui étaient au courant de mes affaires, au courant de ce qu'il y avait dans mon dossier et qui avaient entendu des choses très péjoratives à mon égard. »

\section{Le droit d'être contrainte?}

"...lorsque la personne n'exprime aucune volonté, elle est présumée consentante; si elle proteste, l'admission et le traitement involontaire se justifient. »
Selon Gendreau (2005, p. 160), les psychiatres basent leurs décisions sur la santé de leurs « patients " ${ }^{7}$; ils mettent l'accent sur le besoin qu'a le "malade » de recevoir des soins, alléguant que l'altération de ses facultés constitue un frein à sa guérison. Dans un tel contexte, lorsque la personne n'exprime aucune volonté, elle est présumée consentante; si elle proteste, l'admission et le traitement involontaire se justifient. Cet argument de l'Association mondiale de psychiatrie a pour but de protéger le droit d'administrer un traitement jugé adéquat, sans aucune entrave légale, lorsque le " patient " ne le demande pas ou le refuse. C'est ainsi que la loi sert avant tout à faciliter le travail des experts.

Toujours selon Gendreau (2005), un discours porté par des associations internationales d'usagers affirme à l'instar de Sylvie que les personnes psychiatrisées sont les mieux placées pour évaluer les conséquences d'une médication ou de tout autre traitement. Dans une société libre, pluraliste et démocratique, une personne peut prendre appui sur le droit international pour résister à la coercition exercée par le pouvoir psychiatrique. Il en va du droit à la dignité tout autant que du droit à la différence;s'il n'est pas enchâssé explicitement dans la Déclaration universelle des droits de l'homme, ce dernier est cependant reconnu par l'UNESCO ${ }^{8}$, dans sa Déclaration sur la race et les préjugés raciaux (Article premier, 2) : "Tous les individus et tous les groupes ont le droit d'être différents, de se concevoir et d'être perçus comme tels. [...] »Ainsi, la décision d'accepter ou de refuser un traitement peut reposer sur des critères non médicaux et être pleinement reconnue dans sa valeur, au nom de concepts d'égalité et d'autonomie universellement reconnus. 


\section{"À l'heure où}

\section{l'institution}

psychiatrique ne jouit

plus de droits absolus sur

le destin des personnes,

la coercition constitue un

moyen de procurer des

soins à ceux et à celles

qu'elle cible. »
The right to be different as a source for the right to reject treatment is important for the mentally as well as physically disabled persons. This right is not only encompassed in the Universal Declaration of Human Rights [art. 2 "any status"] but finds specific expression in article 1 of the UNESCO Declaration on Race and Racial Prejudice. (O.I.P.H ${ }^{9}$, cité dans Gendreau, 2005, p. 166)

Davidson (2010) constate que, tout comme le racisme institutionnalisé, la discrimination envers les personnes avec une présumée pathologie de santé mentale a également été institutionnalisée. Les personnes psychiatrisées sont considérées par les autorités comme inférieures et incapables d'assumer leur pleine citoyenneté. À l'heure où l'institution psychiatrique ne jouit plus de droits absolus sur le destin des personnes, la coercition constitue un moyen de procurer des soins à ceux et à celles qu'elle cible.

Alors qu'elle vit en logement, Sylvie est victime d'une agression avec coup et blessure. Cela entraîne une conséquence inattendue : les intervenants du réseau judiciaire débloquent aussitôt des ressources thérapeutiques, ce qui lui permet d'entamer les étapes de sa reconstruction. À titre de victime d'acte criminel, Sylvie aura droit aux services privés d'un psychologue. Des rencontres échelonnées sur quelques mois renforcent son désir de se sortir de l'étau psychiatrique.

Grâce à cette thérapie, elle se sent accueillie, acceptée, reconnue et validée dans ses capacités de prise de décision. Ses efforts pour se libérer de la psychiatrie sont pris au sérieux plutôt que dénigrés. "En quatorze séances, j'ai fait plus de progrès qu'en six ans avec d'autres intervenants! », constate-t-elle. Ces services privés consolident sa décision de se libérer de sa condition psychiatrique qu'elle qualifie d'" industrie du désespoir ". Le tableau suivant résume son parcours de cliente à personne. 
Tableau 1 : «L'industrie du désespoir » : bilan de six année de soins

\begin{tabular}{|l|}
\multicolumn{1}{|c|}{ Conséquences de la psychiatrisation } \\
\hline - Prise de poids importante \\
- Léthargie persistante \\
- Perte de mémoire \\
- Affaiblissement général \\
\hline - Intenses douleurs abdominales \\
- Embolie pulmonaire aiguë \\
- Perte de facultés intellectuelles \\
- Perte de conscience de soi \\
- État semi-végétatif \\
\hline - Barrières à l'emploi \\
- Difficulté d'accéder à un logement décent \\
- Infantilisation \\
- Difficultés conjugales \\
\hline Bilan \\
Le système psychiatrique et celui de l'aide sociale \\
- Alimentent l'insécurité \\
- Cumulent des pratiques opprimantes \\
- Ruinent les rêves de vie.
\end{tabular}

\section{« Je suis allée parler à mon psychiatre et je lui ai dit que je le quittais. "}

"Je suis allée parler à mon psychiatre et je lui ai dit que je le quittais. J'ai quitté aussi le centre de jour.»
D'une façon symbolique, Sylvie choisit le début de l'année 2012 pour marquer une nouvelle étape dans sa vie. Depuis le moment où elle affirme ses choix, elle reprend confiance en ses capacités et en la possibilité d'affirmer ses droits.

"J'ai décidé qu'il fallait que je me distancie du système; j'ai décidé de me renseigner sur mes droits. Je suis allée parler à mon psychiatre et je lui ai dit que je le quittais. J'ai quitté aussi le 
centre de jour. Je ne laisserai pas ma condition me définir.»

Doutant de la capacité de Sylvie de s'en sortir, son ergothérapeute s'oppose à cette décision. Par contre, elle démontre un " intérêt nouveau " pour le vécu quotidien de sa "cliente " et lui pose des questions pressantes sur son mode de vie. Et Sylvie de remarquer :

"Quand j'étais dans le foyer de groupe, alors que je passais des journées entières à regarder la télévision, que j'étais obèse, que je n'arrivais pas à faire une phrase cohérente parce que j'étais complètement dopée, personne ne me demandait si j'allais bien, si je dormais bien, si je mangeais bien, comment j'occupais mes journées...»

\section{Interprétation du récit de Sylvie}

Plusieurs éléments se dégagent du récit de Sylvie. Nous en retenons six qui nous semblent les plus importants, tout en nous centrant sur la perspective structurelle qui nous parait porteuse de pistes d'analyses et d'interventions antioppressives.

\section{Concordance avec d'autres récits}

Les données recueillies dans la démarche de Sylvie et leur analyse concordent avec celles qui émanent d'études ou d'autres témoignages venant de survivants de la psychiatrie (Bassman, 2001; Fisher, 2001; Deegan, 1990) et confirment du même coup

"...l'expérience de

Sylvie suggère qu'il est dangereux de confier à la psychiatrie le meilleur intérêt d'une personne vivant des difficultés personnelles ou familiales. » plusieurs éléments de la pensée antipsychiatrique véhiculée, entre autres, par Szasz (1976), Breggin (1991) et Basaglia (1970). Son plus grand mérite est sans doute de remettre en question le paradigme biomédical. Sylvie constitue une illustration des effets délétères et prolongés que peut provoquer le système psychiatrique chez une personne traversant une période de détresse. En ce sens, il démontre que sa prétention curative et scientifique ne tient pas la route. De plus, l'expérience de Sylvie suggère qu'il est dangereux de confier à la psychiatrie le meilleur intérêt d'une personne vivant 
des difficultés personnelles ou familiales. Dan Fisher (2001) a reçu un diagnostic de schizophrénie. Pourtant, il ne prend aucune médication psychiatrique depuis plus de vingt-cinq ans. Pour lui et pour plusieurs autres, il est inconcevable d'emprisonner les gens dans un stigma d'anormalité chronique; il s'agit d'une tromperie de la part de ceux qui contrôlent l'information psychiatrique.

I have recovered from schizophrenia. If that statement surprises you -- if you think schizophrenia is a lifelong brain disease that cannot be escaped - you have been misled by a cultural misapprehension that needlessly imprisons millions under the label of mental illness [...] I am not an anomaly.Thousands of others have recovered, but are afraid to disclose their past due to the stigma of mental illness. (Fisher, 2001, p. 1)

"Elle entre dans le processus vécu par celles et ceux qui s'identifient comme survivants de la psychiatrie..."

\section{La libération : un processus}

Sylvie exerce à la fois son refus et sa capacité d'empowerment dans un milieu qu'elle décrit comme hostile à ses démarches. Elle entre dans le processus vécu par celles et ceux qui s'identifient comme survivants de la psychiatrie, phénomène qui a surtout été décrit au sein des groupes d'activistes américains de lutte contre les abus de cette dernière. Morrison (2006) met en lumière les étapes qu'une personne traverse dans ce processus de libération. La chercheuse dégage des témoignages recueillis auprès d'expatients ${ }^{10}$ cinq moments de leur évolution personnelle, depuis leur entrée dans le système psychiatrique.Voici les étapes de leur reprise de pouvoir que nous juxtaposons au parcours de Sylvie. 
Tableau 2 : Étapes dans la reprise du pouvoir

\begin{tabular}{|c|l|}
\hline $\begin{array}{c}\text { Étapes de reprise de pouvoir } \\
\text { (Morrison, 2006) }\end{array}$ & \multicolumn{1}{c|}{ Parcours de Sylvie } \\
\hline 1. Entrée dans le système psychiatrique. & $\begin{array}{l}\text { Sylvie est prise en charge par le système de } \\
\text { soins puis amenée à l'hôpital. }\end{array}$ \\
\hline $\begin{array}{c}\text { 2. Désenchantement; sentiment d'avoir été } \\
\text { trahie. }\end{array}$ & $\begin{array}{l}\text { Conditions de vie inacceptables; embolie } \\
\text { pulmonaire; multiples conséquences des } \\
\text { traitements; «Si j'avais su... ». }\end{array}$ \\
\hline $\begin{array}{c}\text { 3. La personne dévie du parcours imposé; } \\
\text { elle utilise des stratégies pour s'en sortir. }\end{array}$ & $\begin{array}{l}\text { Elle décide de quitter le foyer de soins; ten- } \\
\text { tatives d'autonomie; débrouillardise. }\end{array}$ \\
\hline $\begin{array}{l}\text { 4. Elle retrouve des élans de solidarité; elle } \\
\text { reconnait l'oppression qui la brime. }\end{array}$ & $\begin{array}{l}\text { Elle prend conscience de ses droits; elle } \\
\text { quitte son psychiatre; elle rompt avec le } \\
\text { système. }\end{array}$ \\
\hline $\begin{array}{c}\text { 5. Elle s'engage dans des démarches pour } \\
\text { changer le système. }\end{array}$ & $\begin{array}{l}\text { Sylvie s'engage dans des stratégies de résis- } \\
\text { tance, de reprise de ses droits. }\end{array}$ \\
\hline
\end{tabular}

Ainsi, la personne chemine à partir d'un statut d'impuissance jusqu'à une authentique participation sociale, tout comme Sylvie qui résume ainsi son parcours :

"Une chose que j'essaie de faire pour aider les autres, c'est de leur dire : "Essaie de faire ton

"...ce n'est pas normal qu'on te dise : 'Je sais ce qui est bien pour toi et je te l'impose!" " propre bien, parce que ce n'est pas normal qu'on te dise : 'Je sais ce qui est bien pour toi et je te l'impose!" "”

Sylvie constate que presque toutes les personnes qu'elle rencontre au sein des réseaux de services de santé mentale ont subi des traumatismes ou des abus. Elle milite pour qu'on prenne le temps de bien écouter l'histoire des gens. Aujourd'hui, elle attribue un savoir d'expérience aux nombreuses personnes qu'elle croise :

"Je recommande à n'importe qui, lorsqu'il décide de briser les liens avec la psychiatrie, de se renseigner le plus possible sur ses droits parce qu'on n'est pas informés sur nos droits. Beaucoup 
"Si tu cherchais de l'information, c'était accueilli avec hostilité. » de gens pensent ne pas avoir de droits. Il faut vraiment qu'on se donne la peine d'aller vérifier. Mes intervenants ne le prenaient pas du tout que je fasse de la recherche sur les médicaments. Si tu cherchais de l'information, c'était accueilli avec hostilité.»

Diverses conférences et formations l'aident à se reconstruire, comme des ateliers sur le budget, sur la saine alimentation ou sur un programme d'exercices. Sa ligne téléphonique personnelle sert de relais pour de l'aide entre pairs; ce faisant, elle développe une solidarité et une complicité avec d'autres personnes en situation similaire.

\section{Vies perdues ou espoirs retrouvés? "Si j'avais su! »}

À force de détermination, Sylvie a trouvé la force de poser un geste décisif. Il lui aura fallu plus de six ans et des séquelles graves pour en arriver là. Elle en dégage une leçon :

«Si j'avais su qu'à partir de ce moment-là,j'allais être pauvre, je ne pourrais pas travailler plus de quatre heures par semaine, je ne pourrais pas vivre en couple, je ne pourrais pas avoir d'enfants, je ne me serais pas embarquée là-dedans. Je serais partie et j'aurais accepté de l'aide de mes oncles qui demeurent à l'extérieur de la province.»

"Son histoire illustre les rapports de pouvoir et d'oppression exercés par une structure qui prétend trouver en se basant sur un diagnostic médical une explication rationnelle à la souffrance humaine. »
Sylvie refait sa vie tout en étant soucieuse d'aider les personnes confrontées à des défis semblables à ceux qu'elle a vécus. Son histoire illustre les rapports de pouvoir et d'oppression exercés par une structure qui prétend trouver en se basant sur un diagnostic médical une explication rationnelle à la souffrance humaine.

\section{S'attarder au vécu de la personne: perspectives de libération}

Un nombre grandissant de recherches (Provencher, 2007; Rodriguez, Corin et Guay, 2000; Rodriguez, Corin et Poirel, 
"Accueillir et reconnaitre la parole et le potentiel des personnes psychiatrisées est essentiel pour comprendre l'expérience vécue."
2001; Rodriguez et Poirel, 2007; Corin, Poirel et Rodriguez, 2011; Unzicker,Wolters et Robinson, 2000) s'intéressent au point de vue des personnes pour faire contrepoids à une perception de leur réalité faussée en raison du stigma généré par des experts du paradigme médical. À titre d'exemple, une recherche qualitative de Davidson (2003) vise à redéfinir l'expérience de la schizophrénie en privilégiant une démarche inspirée par Husserl (1970) et l'approche phénoménologique. Davidson montre qu'il est possible de rester hors de la "maladie " et que celle-ci ne définit pas l'identité de ceux et de celles à qui un diagnostic a été apposé. Accueillir et reconnaitre la parole et le potentiel des personnes psychiatrisées est essentiel pour comprendre l'expérience vécue.

Dans un tel contexte, accorder de la crédibilité au témoignage de Sylvie permet de repenser ce qui est présenté de prime abord comme un problème de "maladie mentale ". Sylvie a dû défier ses diagnostics et trouver par ses propres moyens des solutions de rechange à un système qui la maintenait dans l'impuissance. Qui plus est, son expérience narrative lui a permis de valider certaines décisions courageuses. En cela, elle fait suite à d'autres expériences narratives menées aux États-Unis par Morrison (2006) qui démontrent à quel point le fait d'accréditer l'expérience des personnes va beaucoup plus loin que l'interprétation d'une étiquette stigmatisante. C'est une opportunité de travailler avec la personne d'une façon non opprimante tout en soutenant sa quête de justice et d'autodétermination.

Acknowledging participant narratives allows a fuller understanding of issues encountered in their relations with mental health professionals. A thoughtful and informed recognition of their experience, more than wordplay with discrediting labels, provides an opportunity to understand, support and work with their efforts to find justice, self-determination, and non-oppressive helping alternatives. (Morrison, 2006, p. 4) 
"Le discours clinique assume qu'il lui appartient d'adapter son comportement à la norme sociale. " «...un individu seul peut transformer une situation de déviance ou de marginalité en un potentiel d'actions porteur d'une influence déterminante sur le système auquel il s'oppose. "

\section{Une situation individuelle, une analyse politique}

Il va de soi qu'une analyse politique découle de cette narration, alors que les difficultés et défis structurels auxquels Sylvie a fait face semblent avoir échappé aux intervenants. Les politiques sociales et les idéologies véhiculées dans un tel contexte deviennent pénalisantes pour des populations minoritaires, déjà infériorisées et vivant dans des situations opprimantes. Attribuer la responsabilité à la personne devient alors une des techniques privilégiées. Le discours clinique assume qu'il lui appartient d'adapter son comportement à la norme sociale. Toute dérogation est vite considérée comme un vice de fonctionnement dans son processus de socialisation, comme l'indique Mullaly (1993, p. 135) : «If a person does not behave in ways expected of, say, a parent, a wageearner, or a law-abiding citizen, it is assumed that something went wrong in that person's socialization process."

Pourtant, il arrive qu'une position conflictuelle provoque un renversement de situation à l'avantage de la personne ciblée jusque-là comme déviante.

\section{Le refus : de la déviance à la dissidence}

«Dans le modèle d'influence sociale que je propose, la création des conflits est une condition nécessaire. Au cours des années, le conflit gagne en intensité et en efficacité.» (Moscovici, 2010, p. 262)

Même dans une apparente absence de pouvoir, un individu seul peut transformer une situation de déviance ou de marginalité en un potentiel d'actions porteur d'une influence déterminante sur le système auquel il s'oppose. Par exemple, Sylvie prend une position claire face au pouvoir psychiatrique. Elle affirme son opposition d'une manière catégorique. La singularité de son refus pourrait se transformer en dissidence si elle persévère et persiste dans un comportement consistant. Il s'agit de la théorie des « minorités actives » telle qu'élaborée par Moscovici (1979)" ${ }^{11}$ qui consiste à provoquer un conflit visant une crise de légitimité 
d'un système opprimant, étant ici celui des systèmes de services reliés à la psychiatrie institutionnelle.

Cette théorie de l'influence sociale a le mérite d'apporter un éclairage additionnel sur une expérience qui se poursuit, dont nous ne connaissons pas encore l'issue. Jusqu'où ira l'influence de Sylvie? Quels en seront les impacts sur d'autres personnes psychiatrisées confrontées à des réalités semblables? Quelle sera la réponse des pouvoirs publics à des gestes prenant la forme d'une dissidence plus formelle? Apparentée à une majorité opprimante, la réaction du système psychiatrique se cantonnera-t-elle dans l'une des trois positions identifiées par Moscovici quand il avance que « [c]onfrontée à un conflit qu'elle ne veut pas et qui touche à son nerf principal, toute majorité choisit d'habitude entre trois réactions possibles : l'ignorance, le compromis ou l'exclusion." (2010, p. 255)?

\section{Bilan de l'expérience : utilité de l'analyse structurelle}

Le témoignage de Sylvie nous convie à réfléchir tant sur le rôle des personnes ayant vécu la psychiatrie ou d'autres formes d'oppression que sur l'aide potentielle qu'offre l'analyse structurelle pour nous amener à comprendre ce genre de parcours et à appuyer efficacement toute démarche de retour à une vie satisfaisante. Voici à ce propos trois dimensions qui se dégagent de cette étude de cas :

\section{(1) L'analyse structurelle servant à décoder certaines facettes du récit}

Dans une institution où le savoir biomédical prend toute la place, l'analyse privilégiée ici permet de s'en distancer au profit d'une perspective de libération. Ce parcours témoigne des éléments associés aux principes d'une approche structurelle associée à une perspective antipsychiatrique. En ce sens, les propos de Sylvie confirment le constat rapporté par LeBlanc et St-Amand (2008, p. 252) : «Dans bien des cas, les soi-disant ressources en santé mentale interviennent sans tenir compte de l'opinion, de l'expérience et de l'expertise des personnes qu'elles traitent.» 
«...les institutions

sociales assurent une régulation visant le renforcement de la structure économicopolitique. "
Selon une grille d'analyse structurelle, l'organisation du système de services sociaux maintient l'ordre dans une société capitaliste fondée sur le profit, le patriarcat et la propriété privée. De leur côté, les institutions sociales assurent une régulation visant le renforcement de la structure économicopolitique (Moreau et Leonard, 1989, p. 24).

Rappelons quelques jalons historiques de l'éclosion de l'approche structurelle. Au cours des années 1950 et 1960, les promesses de l'État-providence laissent croire à une prospérité accessible à toutes et à tous. Comme le souligne Mullaly (2007), dans une telle conjoncture, les problèmes individuels sont renvoyés à des carences personnelles ou familiales, idées soutenues par la théorie freudienne et les approches psychosociales. Mais déjà en 1951, la théorie générale des systèmes de Parsons remet en question le modèle de causalité linéaire inspiré de ce modèle médical dominant. L'étude de "patterns " relationnels des humanistes remet déjà en question les catégories diagnostiques. Cela jette un doute sur les diagnostics de maladie mentale, mais sans nécessairement tenir compte des impacts du sexisme, du racisme, de l'exploitation économique et des autres variables structurelles. Bien que ces théories négligent le contexte politique et social, ainsi que le poids des institutions répressives, elles créent néanmoins une brèche dans l'hégémonie du paradigme médical en santé mentale. "The humanistic theories of Maslow (1968; 1970) and Rogers $(1951 ; 1967)$ among others, redefined long accepted concepts of mental health, seriously questioning the entire diagnostic categories that had underpinned clinical social work practice.» (Moreau et Leonard, 1989, p. 5)

Vers la fin des années 1960, on assiste à une montée de contestation en Occident. C'est l'émergence d'une contre-culture basée sur des valeurs de paix, d'amour et de solidarité, en réaction aux figures oppressives des institutions dominantes. Des théories nouvelles expliquent la souffrance humaine et les déséquilibres personnels par l'analyse des conditions sociales. Des principes marxistes, féministes (Millet, 1971) et antipsychiatriques (Szasz, 1976) alimentent le discours sociologique qui propose que ce soit la société qui est malade et non l'individu. 
"Lorsque des travailleurs sociaux et d'autres intervenants professionnels aident les personnes à s'adapter à leur situation en individualisant et en psychologisant leurs problèmes, ils s'éloignent alors de la véritable mission du service social. »

\section{"Le récit de Sylvie} remet en cause le système de l'aide sociale ainsi que les réseaux publics et privés de soins et d'hébergement."
L'approche structurelle est également associée au concept d'anomie de Durkheim : " [lequel] décrit un état de désintégration sociale né de la division sociale croissante du travail où les contacts sociaux entre travailleurs sont réduits, ce qui empêche toute relation sociale satisfaisante" (Buchholtz, 1992, p. 160). Dans un tel contexte et de prime abord, une perspective structurelle remet en question les conditions économiques, sociales et politiques et explique la création ou l'aggravation du problème ou les obstacles à sa résolution. Lorsque des travailleurs sociaux et d'autres intervenants professionnels aident les personnes à s'adapter à leur situation en individualisant et en psychologisant leurs problèmes, ils s'éloignent alors de la véritable mission du service social (Moreau et Leonard, 1989)

Si les pratiques institutionnelles contribuent à perpétuer des problèmes humains et sociaux, une approche structurelle met en évidence les conditions politiques qui empêchent les gens de se maintenir dans un état de santé satisfaisant. Lorsqu'une personne agit d'une manière différente, dérangeante ou inappropriée par rapport aux normes sociales en vigueur, la société intervient sur l'individu ou la famille via ses institutions pour qu'elle retrouve son fonctionnement dit normal. C'est ce que Mullaly (1993) désigne comme les effets du néoconservatisme, en riposte à l'approche clinique qui pose sa loupe sur les perturbations d'ordre personnel ou familial.

Le récit de Sylvie remet en cause le système de l'aide sociale ainsi que les réseaux publics et privés de soins et d'hébergement. Ces «partenaires» basent leurs interventions sur l'idée de la responsabilité individuelle. Ils ne tiennent pas compte des failles et des contradictions d'ordre social ou politique, ou encore des limites imposées par la pauvreté et le manque de ressources, éléments sur lesquels une approche structurelle travaille de façon nécessaire. Accompagnée par des intervenants du milieu ou par ses propres moyens, la personne mobilise ses forces et celles de ses pairs afin de s'extraire de sa condition. Voilà ce qui nous amène à proposer une vision structurelle à partir de laquelle Sylvie peut agir sur son contexte d'aliénation. On se situe ici dans une perspective de travail social inspirée par Jane Addams (Davidson, 2010; St-Amand, 
2011). Moreau et Leonard (1989, p. 7) exposent les différentes influences qui ont mené au développement des approches structurelles en travail social en rappelant que certains courants sociologiques ont remis en question des pratiques sociales élitistes, soit celles qui servaient d'abord les intérêts de ses promoteurs, au détriment des besoins des personnes souffrantes : « Structural social work therefore links direct work with individuals and families to work with the groups to which they belong. Its main objective is to join organizational and political action to individual action.»

Pour sa part, Mullaly (2007) insiste sur la nécessité de prendre conscience des différentes formes d'aliénation. L' « auto-aliénation » implique une distance dans sa capacité de ressentir ou d'être en contact avec son corps, ses sens, sa capacité de penser ou de percevoir la réalité sociale autour de soi. L'« aliénation provenant d'autrui » implique d'être vu par autrui en tant qu'objet lié à une fonction et non en tant qu'être humain à part entière. Ces deux formes d'aliénation s'observent clairement à l'étude de la démarche de Sylvie. Ses conditions d'aliénation sont étroitement associées aux situations d'oppression décrites par Mullaly (2007, p. 253). Ce qui détermine l'oppression, ce n'est pas d'abord une condition individuelle, mais bien le fait d'appartenir à un groupe particulier ou à une catégorie de personnes identifiées dans un contexte relationnel - des personnes étiquetées de "malades mentales "

«Ce n'est pas le problème qui opprime, mais bien le lien d'aliénation avec ceux qui y prennent appui pour imposer leurs modes d'action."
-, alors que de l'autre côté, un groupe détient du pouvoir, de l'influence et des privilèges. Ce n'est pas le problème qui opprime, mais bien le lien d'aliénation avec ceux qui y prennent appui pour imposer leurs modes d'action. Selon Mullaly (2007), l'oppression liée à l'exclusion et au maintien dans la pauvreté est structurelle

does not occur today through some coercive rule of law or because of the evil intentions of a dominant group. It occurs through the systemic constraints on subordinate groups that take the form of unquestioned norms, behaviours, and symbols and in the underlying assumptions of institutional rules. In other words, modern-day oppression is structural. (p. 261) 
"Plutôt que de provoquer la mort par des actes violents, l'oppression structurelle provoque une mort lente et prématurée, en toute impunité pour ceux qui en sont les instigateurs. "
Cette forme de violence institutionnelle est largement acceptée; elle devient même partie intégrante de ce qui est généralement admis comme la condition "normale " des groupes qui la subissent. Pour Holmes et Murray (2012), le fait de présumer que les personnes psychiatrisées ne veulent pas changer et celui de centrer les interventions sur les symptômes et les comportements en négligeant les causes socioéconomiques, politiques et environnementales les ayant menées jusque-là peuvent en soi être analysés comme une violence : alors que le modèle biomédical est devenu incontrôlable, non éthique et inefficace, il produit une iatrogénèse clinique et sociale. Une fois identifiée, cette violence permet d'en comprendre les ramifications jusque dans les rapports de domination que met en lumière l'expérience de Sylvie. Plutôt que de provoquer la mort par des actes violents, l'oppression structurelle provoque une mort lente et prématurée, en toute impunité pour ceux qui en sont les instigateurs. "Bulhan's definition of violence links it with structural oppression : "Violence is any relation, process, or condition by which an individual or a group violates the physical, social, and/or psychological integrity of another person or group.” " (Mullaly, 2007, p. 271)

Ces constatations invitent le chercheur ou l'intervenant à se questionner sur la voie possible à prendre pour sortir de cette contradiction. À la base, une lecture de la société fondée sur des analyses critiques conditionne l'approche sur laquelle la personne peut prendre appui dans son processus de reconstruction.

Leonard contends that there are three requirements or undertakings of a critical theory : (1) it must locate the sources of domination in actual social practices; (2) it must present an alternative vision (or at least an outline) of a life free from such domination; and (3) it must translate these tasks in a form that is intelligible to those who are oppressed in society. (Mullaly, 1993, p. 142)

Toute intervention qui vise à nommer, localiser, neutraliser et remplacer la source oppressive de domination s'inscrit en continuité avec les approches critiques et l'intervention 
"...une histoire psychiatrique qui nous invite à dépasser l'indignation et à déboucher sur une analyse politique $d u$ pouvoir. »
structurelle.Voilà à notre avis un lien à explorer en réaction à une histoire psychiatrique qui nous invite à dépasser l'indignation et à déboucher sur une analyse politique du pouvoir.

\section{(2) L'analyse structurelle et les perspectives antipsychiatriques}

La pensée antipsychiatrique s'est surtout affirmée à partir des années 1970, grâce aux psychiatres Cooper (1970) et Laing (1972) en Angleterre et à Jean Oury et Félix Guattari (1977) en France. En Italie, Franco Basaglia et Giovanni Jervis ${ }^{12}$ ont procédé à une fermeture massive d'hôpitaux psychiatriques en mettant de l'avant un véritable mouvement politique qui dépasse l'institution psychiatrique comme telle. «Non seulement l'hôpital psychiatrique est dénoncé, mais en plus, cette remise en cause est insérée dans la contestation de la société tout entière. C'est le système capitaliste qui est critiqué.» (Lesage de La Haye, 2006, p. 43$)^{13}$

Aux États-Unis, le psychiatre Thomas Szasz, (1976; 2010), surnommé "le père de l'antipsychiatrie ", remet en question toute la psychiatrie coercitive alors que Peter Breggin (1991; 1999) milite depuis plus de trente ans contre la toxicité de la psychiatrie chimique. Ce dernier montre que l'État et la famille représentent des institutions aliénantes utilisant la psychiatrie à des fins de contrôle social. Le discours est clair : la société, et non l'individu, doit être soignée. L'hôpital rend malade et provoque un état prolongé, voire permanent, d'incapacité, de ségrégation et d'isolement social (Basaglia et Basaglia Ongaro, 1980). Les idées fondatrices de l'antipsychiatrie traversent les époques, nourrissent les débats ${ }^{14}$ et contribuent encore aujourd'hui à une remise en question du paradigme biopsychiatrique et de son pouvoir oppressif. À l'exemple de Sylvie, les perspectives antipsychiatriques demeurent importantes à rappeler ici dans ce contexte de refus et de réappropriation.

\section{(3) L'analyse structurelle et l'intervention}

Comment intervenir dans l'optique d'une pratique sociale qui tienne compte de cette analyse du récit de Sylvie? Selon 


\section{"Comprendre le} contexte social, politique et économique dans lequel évolue la personne, ce qui a engendré la crise qu'elle doit gérer, est la première obligation de l'intervenant structurel. "

"Plutôt que de s'attaquer aux problèmes structurels, l'intervention met l'accent sur l'adaptation et le contrôle social."
Mullaly (2007), c'est à la combinaison de facteurs personnels et sociaux qu'il y a lieu de s'attaquer pour viser une transformation individuelle grâce à des changements structurels. Cela implique de travailler à la fois avec les personnes, les familles et les instances politiques ou institutionnelles pour modifier les rapports sociaux et le lien social.

Selon l'expérience de Sylvie, les pratiques institutionnelles et les interventions professionnelles retournent sur l'individu les conditions de son oppression structurelle. Comprendre le contexte social, politique et économique dans lequel évolue la personne, ce qui a engendré la crise qu'elle doit gérer, est la première obligation de l'intervenant structurel. Qui plus est, selon Morley et Macfarlane (2010), le travail social implique une prise de position en faveur de la justice sociale, de l'équité et des droits de la personne. L'analyse structurelle représente ainsi la pierre angulaire de cette discipline. Un appui à la psychiatrie trahit la mission du service social. Voilà pourquoi on se doit d'offrir une solution de rechange critique aux pratiques oppressives dont Sylvie est l'une des nombreuses victimes.

Dans un tel contexte, Reich (2011) croit que les praticiens du travail social doivent repenser leur rôle et leur relation à l'État, à la communauté, aux utilisateurs de services et aux forces du marché, tant dans leurs interventions individuelles que dans leurs actions collectives. Il décrit la société contemporaine comme assujettie aux buts et aux valeurs des forces institutionnelles néolibérales, alors que les pratiques en travail social ont tendance à se concentrer sur la transformation de l'individu, les approches comportementales et la coercition. Plutôt que de s'attaquer aux problèmes structurels, l'intervention met l'accent sur l'adaptation et le contrôle social. Selon Moreau et Leonard (1989), par contre, il s'agit de développer une « conception dialectique » de la pratique sociale, touchant à la fois les contradictions, les luttes et les conflits affectant la personne dans son milieu, travaillant dans le système et hors de celui-ci, gardant une distance critique vis-à-vis des aspects oppressifs de la pratique institutionnelle.

La situation vécue par Sylvie illustre un modèle d'intervention institutionnelle intégré, porteur de lourds préjudices, impliquant 
le réseau de la santé, l'appareil d'État et le secteur privé. Le pouvoir psychiatrique passe le relais à des ressources privées d'hébergement. Le pharmacien local, agent d'affaires de l'industrie du médicament, est étroitement associé à la prise en charge de la personne psychiatrisée. Le niveau de contrôle exercé par la psychiatrie va jusqu'à la rendre entièrement incapable de prendre soin d'elle-même, la plaçant dans un état de ségrégation, comme le décrit Davidson (2003). Le modèle médical perpétue ainsi sa logique même à l'extérieur des lieux institutionnels. "[T]hese same institutional structures and attitudes can be found operating at a more implicit, but yet powerful, level in the impoverished lives of hundreds of thousands of individuals now living in the community. » (Davidson, 2003, p. 36)

De l'analyse des causes structurelles de la souffrance psychique émerge la question suivante : est-il possible d'intervenir dans une

"Cette lutte que Sylvie mène tous les jours sert d'illustration des multiples blessures subies par un système qui a presque réussi une mort en douce de ses rêves et de son potentiel.» perspective de lutte pour l'émancipation des personnes et des groupes victimes des structures qui les prennent en otages? La lutte contre une obésité iatrogénique de plusieurs années illustre les contradictions du système : ce problème - pour ne pas dire ce "détail» — est laissé à l'entière responsabilité de Sylvie, à sa charge et à ses frais. Cette lutte que Sylvie mène tous les jours sert d'illustration des multiples blessures subies par un système qui a presque réussi une mort en douce de ses rêves et de son potentiel.

\section{En guise de conclusion : Et le service social dans tout ça?}

Le service social peut contribuer à perpétuer les problèmes sociaux ou encore à les enrayer; voilà les deux grandes tendances que nous pouvons observer dans la profession depuis sa création (St Amand, 2011). Certains praticiens choisiront de confronter les contradictions du système alors que d'autres s'efforceront d'encourager les gens à s'adapter à des structures sociales injustes et opprimantes. Rappelons que ce choix se posait déjà aux débuts de la profession au $19^{\mathrm{e}}$ siècle; alors que les approches de casework se développaient sous l'influence de Mary Richmond, Jane 
"Pour toutes les personnes qui, restées dans l'ombre de l'anonymat et de la peur, cachées derrière le mur des rapports thérapeutiques confidentiels, ne voient pas de moyens de s'en sortir, Sylvie représente une lueur d'espoir. "
Addams ${ }^{15}$ s'engageait dans la promotion des droits des femmes et des personnes immigrantes, militait pour de meilleures conditions de vie et de logement. La maison Hull House qu'elle avait fondée devint un carrefour important pour les penseurs progressistes. Par des interventions inclusives, elle donnait l'exemple d'une communauté engagée (St-Amand, 2011). Son nom reste associé à celui d'une pionnière de la vision structurelle du service social.

L'analyse structurelle se fonde sur l'expérience de personnes et de groupes dont le parcours s'apparente à celui de Sylvie; le renversement des rapports de pouvoir ressort clairement de son parcours. Et bien que la dimension genre ne soit pas explicitement identifiée, les rapports hommes-femmes auxquels nous invite une analyse structurelle sont bien présents dans sa relation avec son psychiatre en particulier. Enfin, les références à « l'Importée » font ressortir la vulnérabilité des personnes en provenance d'autres ethnies. En somme, nous pouvons identifier à l'aide d'une grille structurelle plusieurs relations de pouvoir qui se traduisent sur le plan de la classe sociale, du genre et de l'ethnie en particulier. De plus, une analyse antipsychiatrique ajoute un élément à ce récit, car nous estimons que la remise en question de la psychiatrie s'arrime ici avec une analyse structurelle.

Cette initiative de recherche et l'analyse de contenu qui en émerge illustrent la nécessité de poursuivre l'étude des parcours individuels des gens qui luttent pour leur survie et leurs droits et qui finissent souvent par accepter un diagnostic psychiatrique avec ses conséquences. Pour toutes les personnes qui, restées dans l'ombre de l'anonymat et de la peur, cachées derrière le mur des rapports thérapeutiques confidentiels, ne voient pas de moyens de s'en sortir, Sylvie représente une lueur d'espoir. Son récit met les chercheurs sur la piste d'initiatives amorcées par plusieurs mouvements et associations d'usagers. Le message est clair : il est possible de "devenir de véritables citoyens, capables de penser, d'exister à l'extérieur du système psychiatrique " (LeBlanc et St-Amand, 2008, p. 222). Sylvie sert d'exemple, sinon de modèle, tant pour ces personnes qui luttent au quotidien que pour les chercheurs et intervenants qui s'intéressent à une véritable santé mentale. 
"Il s'agit là d'un engagement véritable à modifier tant les conditions personnelles que sociales qui contribuent à psychiatriser non seulement les gens vulnérables, mais également notre société. "
Toutes et tous veulent comprendre la complexité des situations des individus dont la souffrance devient essentielle au maintien d'un système opprimant présenté sous couvert d'aide thérapeutique. Pour comprendre Sylvie et les milliers de personnes qu'elle représente, une analyse structurelle des divers facteurs d'oppression reste incontournable. À l'image du Refus global de Borduas, le refus de cette "aide " et des conséquences qu'elle entraîne constitue la première étape d'une authentique reconstruction de soi. Il s'agit là d'un engagement véritable à modifier tant les conditions personnelles que sociales qui contribuent à psychiatriser non seulement les gens vulnérables, mais également notre société.

\section{Notes}

1 Cette démarche s'inscrit dans le cadre d'un projet canadien, Le champ francophone de la désinstitutionnalisation en santé mentale : enjeux sociohistoriques, normes et pratiques, 1920-1980 (THIFAULT, et collab.), qui vise à évaluer les pratiques de désinstitutionnalisation en Ontario, au Québec et au Nouveau-Brunswick.

2 Mots clés : Zyprexa et diabète $:$ http://www.votre-sante.com/news.php?dateedit=1304454261

3 (Zyprexa) : association soupçonnée avec une embolie pulmonaire, documenté en 2003, 2004, 2005 : http://www.hc-sc.gc.ca/dhp-mps/medeff/bulletin/carn-bcei_v15n1-fra.php\#a7

4 Voir La Presse, 8 juin 2011, http://www.lapresse.ca/vivre/sante/201106/08/01-4407287-lespilules-contraceptives-yaz-et-yasmin-dangereuses-pour-la-sante.php

5 Risque accru de formation de caillots sanguins associé aux contraceptifs oraux Yasmin et Yaz : Santé Canada : http://www.hc-sc.gc.ca/ahc-asc/media/advisories-avis/_2011/2011_164-fra. php «Au Canada, aux États-Unis et en France, des contraceptifs oraux de dernière génération sont montrés du doigt par des femmes qui ont contracté de graves problèmes de santé. Les requêtes judiciaires s'accumulent notamment contre la firme Bayer..." http://www.lapresse. ca/actualites/quebec-canada/sante/201302/02/01-4617750-des-pilules-

contraceptives-de-bayer-au-banc-des-accuses.php

$6 \quad$ L'histoire de la clozapine est mal connue. Elle a été introduite en Amérique du Nord en 1989 par la compagnie Sandoz, qui a fait un grand tapage publicitaire autour de cette prétendue innovation. Or, la clozapine existait déjà en Europe où elle était prescrite depuis le début des années 1960. En 1972, son usage avait déjà été remis en question sur le continent européen parce qu'elle avait causé vingt décès en produisant chez des patients une chute vertigineuse des globules blancs (agranulocytose), un de ses effets secondaires les plus redoutables. En 1993, on recensait aux États-Unis un peu plus de 11500 personnes qui prenaient de la clozapine. De ce nombre, 73 ont développé une agranulocytose, réaction qui fut fatale dans deux cas. À cause de cette conséquence toxique très grave, la clozapine n'est prescrite que si la personne concernée peut subir des prises de sang bimensuelles. La clozapine comporte également un risque important 
de convulsions (entre $2 \%$ et $10 \%$ ) et ce risque augmente avec la dose (Cohen, Cailloux-Cohen et AGIDD-SMQ, 1995, p.184-185).

7 Le terme "patient " est mis en italique pour respecter à la fois la formulation de Gendreau (2005) et notre souci de ne pas utiliser sans réserve une désignation infériorisante et infamante qui aliène, opprime la personne de son statut de citoyen. (Voir St-Amand, 2011, p. 125)

8 UNESCO Déclaration sur la race et les préjugés raciaux 27 novembre 1978, http://portal. unesco.org/fr/ev.php-URL_ID=13161\&URL_DO=DO_TOPIC\&URL_SECTION=201. html

9 Organisation internationale des personnes handicapées, Disabled Peoples International, http:// www.dpi.org

10 Désigné par le vocable c/s/x : consumer/survivor/ex-patient

11 Nous avons consulté la version mise en ligne par J. M. Tremblay (2010) sur le site : Classiques des sciences sociales, http://classiques.uqac.ca/

12 Franco Basaglia et Giovanni Jervis sont des psychiatres qui ont été les principaux initiateurs de la réforme institutionnelle de la psychiatrie italienne à partir des années 1970, notamment à l'asile de Gorizia et à celui de Trieste. À ce titre, ils ont été les porte-paroles d'une antipsychiatrie de nature politique et antioppressive.

13 Cité dans Lien Social, réf. du 22 février 2013, http://www.liensocial.com/article.php3?id_ article $=1285 \&$ id_groupe $=7$

14 Voir notamment : Actualités et documentation sur MindFreedom, Paula Caplan sur Mad in America, ainsi qu'une critique plus mitigée de la psychiatrie sur Postpsychiatry.com

15 Elle obtient le prix Nobel de la paix en 1931.

\section{Bibliographie}

BASAGLIA, Franco (1970). L'institution en négation : rapport sur l'hôpital psychiatrique de Gorizia, Paris, Éditions du Seuil, $281 \mathrm{p}$.

BASAGLIA, Franco, et Franca BASAGLIA ONGARO (1980). Les criminels de paix : recherches sur les intellectuels et leurs techniques comme préposés à l'oppression, Paris, Presses Universitaires de France, $332 \mathrm{p}$.

BASSMAN, Ronald (2001). "Whose reality is it anyway? Consumers/survivors/ex-patients can speak for themselves ", Journal of Humanistic Psychology, Vol. 41, No 4, p. 11-35.

BERTAUX, Daniel (2005). Le récit de vie : l'enquête et ses méthodes, Paris, Armand Colin, 126 p.

BORDUAS, Paul-Émile (1948). Le refus global, réf. du 21 décembre 2012, http://fr.wikisource.org/wiki/Refus_global

BRACKEN, Pat (2012). "Postpsychiatry - Reaching beyond technological paradigm in mental health care ", [Conférence vidéo (1 h 46 min 50 s)], réf. du 22 février 2013, http://postpsychiatry.com/?page_id $=35$

BRACKEN, Pat (2012). "Postpsychiatry: Reaching beyond the technological paradigm in mental health care ", [Diaporama de presentation], réf. du 22 février 2013, http://www.eksistentielpsykologi.dk/wp-content/uploads/2012/06/Slides-for-Copenhagen. ppt 
BRACKEN, Pat (2012). "Postpsychiatry: Reaching beyond the technological paradigm in mental health care ", [Notes de conference], réf. du 22 février 2013, http://www.eksistentielpsykologi. $\mathrm{dk} /$ wp-content/uploads/2012/06/Talk-for-Copenhagen1.doc

BREGGIN, Peter R., et David COHEN (1999). Your drug may be your problem: How and why to stop taking psychiatric drugs, Reading, Mass, Perseus Books, 273 p.

BREGGIN, Peter R. (1991). Toxic psychiatry: Why therapy, empathy, and love must replace the drugs, electroshock, and biochemical theories of the "new psychiatry", New York, St. Martin's Press, $480 \mathrm{p}$.

BUCHHOLTZ, Anke (1992). "Les conceptions quotidiennes de la maladie psychique - Théories subjectives des proches ", dans Uwe Flick (dir.), La perception quotidienne de la santé et de la maladie-théories subjectives et représentations sociales, collection Santé, sociétés et culture, Paris, l'Harmattan, p. 156-176.

CAPLAN, Paula (2012). Psychiatric survivors speak up: Harm from psychiatric diagnosis, and a start on solutions, [Conférence vidéo (01:06:22)] dans Mad in America, Science, Psychiatry and Community, réf. du 12 mars 2013, http://www.madinamerica.com/2013/02/psychiatric-survivors-speakup-harm-from-psychiatric-diagnosis-and-a-start-on-solutions/

CARLAT, Daniel (2010). Unhinged:The trouble with psychiatry-a doctor's revelations about a profession in crisis, New York, Free Press, 262 p.

COHEN, David, Suzanne CAILLOUX-COHEN et AGIDD-SMQ (1995). Guide critique des médicaments de l'âme, Montréal, Éditions de l'homme, 409 p.

COOPER, David (1970). Psychiatrie et antipsychiatrie, Paris, Éditions du Seuil, réf. du 22 novembre 2012, myreader.toile-libre.org/uploads/My_4eda1aa27541a.pdf

CORIN, Ellen, Marie-Laurence POIREL et Lourdes RODRIGUEZ (2011). Le mouvement de l'être : paramètres pour une approche alternative du traitement en santé mentale, Montréal, Presses de l'Université du Québec, 218 p.

CORNELLIER, Louis (2013). "La folie de la maladie mentale ", Le Devoir, 23 février 2013, page C3, Numéro de document : news·20130223·LE·2013-02-23_371519, réf. du 23 février 2013, http://www.ledevoir.com/culture/livres/371519/la-folie-de-la-maladie-mentale

CYR, Emmanuelle (2010). De l'action collective à l'emprise institutionnelle : l'apport des psychiatrisés dans la vision du rétablissement en santé mentale, Mémoire de maitrise, Université d'Ottawa, École de service social.

DAOUST-BOISVERT, Amélie (2013). "Un toit pour se sortir de la rue ", Le Devoir 14 juillet 2012, réf. du 22 novembre 2012, http://www.ledevoir.com/societe/sante/354607/un-toit-pour-sesortir-de-la-rue.

DAVIDSON, Larry (2003). Living outside mental illness - Qualitative studies of recovery in schizophrenia, New York, University Press, $227 \mathrm{p}$.

DAVIDSON, Larry (2010). The roots of the recovery movement in psychiatry, lessons learned, UK, Wiley Blackwell Oxford, 294 p.

DE GAULEJAC Vincent, et Michel LEGRAND (2008). Intervenir par le récit de vie : entre histoire collective et histoire individuelle, Ramonville Saint-Agne, Erès, 335 p.

DEEGAN, Patricia (1990). "Spirit breaking: When the helping professions hurt ", The Humanistic Psychologist, Vol. 18, No 3, p. 301-313.

ERHENBERG, Alain (2010). La société du malaise, Paris, Odile Jacob, 439 p. 
FARMER, Olivier (2012). "Traitement, réadaptation, rétablissement, comment s'y retrouver? ", Conférence d'ouverture, journée Roland-Saucier 2012, Chicoutimi, réf. du 22 novembre 2012, http://www.regroupement.net/journeeRS.htm

FISHER, Daniel (2001). "A look at... Coping with mental illness we've been misled by the drug industry ", The Washington Post, August 19, 2001, p. B03, réf. du 22 février 2013, http://www. power2u.org/articles/recovery/misled.html

GAGNON, Yves-Chantal (2005). L'étude de cas comme méthode de recherche, Montréal, Presses de l’Université du Québec, Montréal, 123 p.

GENDREAU, Caroline (2005). Le droit du patient psychiatrique de consentir à un traitement :élaboration d'une norme internationale, Montréal, Les éditions Thémis, Montréal, 342 p.

GUATTARI, Félix (1980). La révolution moléculaire, Paris, Union générale d'éditions, 400 p.

HERZ, Marcus, et Thomas Johansson (2011). «Critical social work - Considerations and suggestions ", Critical Social Work,Vol. 12, № 1, p. 28 à 45.

HOLMES, Dave, et Stuart J. MURRAY (2012). «A critical reflexion on the use of behaviour modification programmes in forensic psychiatry settings », dans Rudge Holmes et Amélie Perron (dirs.), (Re)thinking violence in health care settings, a critical approach, Burlington (USA), ed.Ashgate, p. 21-31.

HUSSERL, Edmund (1970). The crisis of European sciences and transcendental phenomenology: An introduction to phenomenological philosophy, Evanston, Northwestern University Press, 405 p.

IFE, Jim (2008). Human rights and social work, Toward right based practice, [édition revisée], Sydney, Australie, Cambridge University Press, 230 p.

JERVIS, Giovanni (1973). Psychiatrie et lutte de classes, Strasbourg,Vroutsch, 40 p.

JERVIS, Giovanni (1980). "Crise de la psychiatrie et contradictions institutionnelles ", dans Franco Basaglia et Franca Basaglia Ongaro (1980). Les criminels de paix : recherches sur les intellectuels et leurs techniques comme préposés à l'oppression, Paris, Presses Universitaires de France, p. 227-246.

KAUFMANN, Jean-Claude (2004). L'entretien compréhensif,Ville, Éditions Armand Colin, collection sociologie, $128 \mathrm{p}$.

LAING, Ronald D. (1980). La politique de l'expérience, Paris, Stock, 127 p.

LAING, Ronald D., et David COOPER (1971). Raison et violence : dix ans de la philosophie de Sartre (1950-1960), Paris, Payot, 203 p.

LEBLANC, Eugène, et Nérée ST-AMAND (2008). Osons imaginer : de la folie à la fierté, Moncton (NB), OurVoice/Notre voix, 293 p.

LECOMTE, Tania, et Claude LECLERC (2006). Manuel de réadaptation psychiatrique, Sainte-Foy (Québec), Presses de l'Université du Québec, 461 p.

LESAGE DE LA HAYE, Jacques (2006). La mort de l'asile : histoire de l'antipsychiatrie, Saint-Georgesd'Oléron, Éditions libertaires, 253 p.

MILLETT, Kate (1971). La politique du mâle, Paris, Stock, 521 p.

MINDFREEDOM (2013). " MindFreedom's library of information about mental health, psychiatry, human rights \& alternatives ", réf. du 12 mars 2013, http://www.mindfreedom.org/kb

MINTZES, Barbara, et RÉSEAU CANADIEN POUR LA SANTÉ DES FEMMES (2004). «Des inquiétudes concernant l'innocuité du médicament Diane-35 », réf. du 11 mars 2013, Le Réseau > Été/Automne 2004,Volume 7, No 2/3 http://www.cwhn.ca/fr/node/39961 
MOREAU, Maurice (1987). «L'approche structurelle en travail social : implications pratiques d'une approche intégrée conflictuelle ", Service social,Vol. 36, Nos 2 et 3, p. 227-247.

MOREAU, Maurice, et Lynne LEONARD (1989). Empowerment through a structural approach to social work, a report from practice, Ottawa, Carleton University et École de service social de l'Université de Montréal, 326 p.

MORISSON, Linda J. (2006). "A matter of definition: Acknowledging consumer/survivor experiences through narrative ", Radical Psychology, Vol. 5, réf. du 22 novembre 2012, http://www.radicalpsychology.org/index.html

MORLEY, Christine, et Selma MACFARLANE (2010). "Repositioning social work in mental health: Challenges and opportunities for critical practice ", Critical Social Work, Vol. 11, No 2 p. 46-59.

MOSCOVICI, Serge (2010). Psychologie des minorités actives, Chicoutimi :J.-M. Tremblay, réf. du 22 nov.2012,http://classiques.uqac.ca/contemporains/moscovici_serge/psycho_minorites_actives/ psycho_minorites_actives.pdf

MULLALY, Robert P. (1993). Structural social work: Ideology, theory, practice, Toronto McClelland \& Stewart, $228 \mathrm{p}$.

MULLALY, Robert P. (2007). The new structural social work, Don Mills (Ontario), Oxford University Press, $398 \mathrm{p}$.

OURY, Jean (1976). Psychiatrie et psychothérapie institutionnelle traces et configurations précaires, Paris, Payot, $300 \mathrm{p}$.

PASSERON, Jean-Claude, et Jacques REVEL (dir.) (2005). Penser par cas, Paris, Éditions de l'École des Hautes Études en Sciences Sociales, 292 p.

PINARD Jean-Luc, et Nérée ST-AMAND (2012). Entre l'indignation et la résignation : un espace de réflexion..., [présentation de mai 2012], Montréal, ACFAS.

PINEAU, Gaston, et Marie-Michèle (1983). Produire sa vie : autoformation et autobiographie, Paris, Edilig, 419 p.

PINEAU, Gaston, et Jean-Louis LE GRAND (1993). Les Histoires de vie, Paris, Les Presses Universitaires de France, 285 p.

POIREL, Marie-Laurence, et Ellen CORIN (2011). "Revisiter la notion de traitement à partir de récits de personnes usagères, d'intervenants et de responsables de ressources alternatives en santé mentale : la question de la subjectivité ", Santé mentale au Québec,Vol.36, № 1, p. 115-130.

LA PRESSE CANADIENNE (2011). "Les pilules contraceptives Yaz et Yasmin dangereuses pour la santé? », La Presse, 8 juin 2011, réf. du 22 nov. 2012,

http://www.lapresse.ca/vivre/sante/201106/08/01-4407287-les-pilules-contraceptives-yazet-yasmin-dangereuses-pour-la-sante.php

PROVENCHER, Ysabel (2007). "Aide sociale au Nouveau-Brunswick : le discours des personnes assistées sociales au sujet de leurs rapports avec les gestionnaires de cas », Revue canadienne de politique sociale, $\mathrm{N}^{\circ} 59$, p. 56-75.

REISH, Michael (2011). "Being a radical social worker in reactionary times ", Keynote address to the 25th Anniversary Conference of the Social Welfare Action Alliance, Washington, DC, June 10, 2011, réf. du 22 nov. 2012 http://www.socialwelfareactionalliance.org/reisch_keynote_110610.pdf 
RODRIGUEZ, Lourdes, Ellen CORIN et Lorraine GUAY (2000). «La thérapie alternative : se (re)mettre en mouvement ", dans Yves Lecomte et Jean Gagné, Les ressources alternatives de traitement, Montréal, Regroupement des ressources alternatives en santé mentale du Québec et Santé mentale au Québec, p. 44-94.

RODRIGUEZ, Lourdes, Ellen CORIN et Marie-Laurence POIREL (2001). «Le point de vue des utilisateurs sur l'emploi de la médication en psychiatrie : une voix ignorée ", Revue québécoise de psychologie, Vol. 22, $\mathrm{N}^{\circ}$ 2, p. 1-23.

RODRIGUEZ, Lourdes, et Marie-Laurence POIREL (2007). «Émergence d'espaces de parole et d'action autour de l'utilisation de psychotropes : la gestion autonome de médicaments de l'âme ", Nouvelles pratiques sociales, Vol. 19, № 2, p. 111-127.

SARTRE, Jean-Paul (1960). Critique de la raison dialectique, Paris, Gallimard, 921 p.

ST-AMAND, Nérée (2011). Repenser le service social?, Ottawa, Merriam Print, 324 p.

ST-ONGE, Jean-Claude. (2013). Tous fous? : L'influence de l'industrie pharmaceutique sur la psychiatrie, Montréal, Éditions Écosociété.

SZASZ, Thomas S. (1976). Fabriquer la folie, Paris, Payot, 348 p.

SZASZ, Thomas S. (2010). "Psychiatry, anti-psychiatry, critical psychiatry: What do these terms mean? ", Philosophy, Psychiatry, \& Psychology, Vol. 17, № 3, p. 229-232.

THIFAULT, Marie-Claude, et Nérée ST-AMAND (2010). Demande de recherche au IRSC pour le projet "Le champ francophone de la désinstitutionnalisation en santé mentale : enjeux sociohistoriques, normes et pratiques, 1920-1980 ", Ottawa, Université d'Ottawa, École de service social.

UNZICKER, Rae E., Kate PWOLTERS et Debra ROBINSON (2000). «From privileges to rights: People labeled with psychiatric disabilities speak for themselves ", 2012 National Council on Disability, Washington, DC. réf. du 22 novembre, http://www.ncd.gov/publications/2000/ Jan202000 